\title{
Evaluation of Ablation Patterns Using a Biophysical Model of Atrial Fibrillation
}

\author{
L. DANG,${ }^{1}$ N. Virag,${ }^{2}$ Z. IHARA, ${ }^{1}$ V. JACQUEMET,${ }^{1}$ J.-M. Vesin, ${ }^{1}$ J. SCHLAEPFER,${ }^{3}$ \\ P. RUCHAT, ${ }^{3}$ and L. KAPPENBERGER ${ }^{3}$ \\ ${ }^{1}$ Signal Processing Institute, Swiss Federal Institute of Technology, Lausanne, Switzerland; ${ }^{2}$ Swiss R\&D, Medtronic Europe, \\ Tolochenaz, Switzerland; and ${ }^{3}$ Service of Cardiology, CHUV, Lausanne, Switzerland
}

(Received 19 May 2004; accepted 3 November 2004)

\begin{abstract}
Atrial fibrillation (AF) is the most common form of cardiac arrhythmia. Surgical/Radiofrequency (RF) ablation is a therapeutic procedure that consists of creating lines of conduction block to interrupt AF. The present study evaluated 13 different ablation patterns by means of a biophysical model of the human atria. In this model, ablation lines were abruptly applied transmurally during simulated sustained AF, and success rate, time to AF termination and average beat-to-beat interval were documented. The gold standard Cox's Maze III procedure was taken as reference. The effectiveness of twelve less invasive patterns was compared to it. In some of these incomplete lines (entailing a gap) were simulated. Finally, the computer simulations were compared to clinical data. The results show that the model reproduces observations made in vivo: (1) the Maze III is the most efficient ablation procedure; (2) less invasive patterns should include lines in both right and left atrium; (3) incomplete ablation lines between the pulmonary veins and the mitral valve annulus lead to uncommon flutter; (4) computer simulations of incomplete lines are consistent with clinical results of non-transumural RF ablation. Biophysical modeling may therefore be considered as a useful tool for understanding the mechanisms underlying AF therapies.
\end{abstract}

Keywords-Atrial fibrillation, Catheter ablation, Computer simulation, Maze procedure.

\section{INTRODUCTION}

Atrial fibrillation (AF) is a major medical problem. ${ }^{21}$ Several treatments are available to restore sinus rhythm or to prevent associated stroke: medication, electrical cardioversion, atrial pacing, surgical and catheter ablation. ${ }^{17,18,24}$ The objective of AF ablation is to create lines of conduction block to prevent formation and maintenance of multiple wavelets. The surgical maze ablation was proposed by Cox et al. for patients with AF undergoing open-heart surgery for valve replacement or coronary bypass. ${ }^{4}$ This procedure consists of applying surgical incisions to both atria according to a specific pattern. Although a high success rate was

Address correspondence to Lam Dang, LTS1-ITS-STI, EPFL, 1015 Lausanne, Switzerland. Electronic mail: lam.dang@epfl.ch reported (80-99\%), the results were difficult to reproduce and many clinical complications were observed..$^{20}$

More recently, percutaneous radiofrequency (RF) catheter ablation was proposed as a less invasive alternative to surgical ablation. On the basis of the Maze operation different series of lesion patterns were suggested. ${ }^{4,7,12,20}$ An ideal pattern should be able to prevent $\mathrm{AF}$ with a limited number of ablation lines of minimal length, while allowing for maintenance or recovery of mechanical activity of both atria during sinus rhythm. However, this ideal ablation pattern is still unknown.

The evaluation of different lesion patterns is usually performed in clinical studies or in animal experiments. The main drawbacks of animal experiments lie in the difficulty to technically access the whole atria and in the differences between animal and human anatomy. As a complementary tool, we have developed a biophysical model of AF to assess the efficacy of different patterns of ablation lines. Compared to clinical and animal studies, this approach has the advantage of repeatability under controlled conditions.

In this paper, we first present the result of simulations involving the gold standard Cox's Maze III procedure. In the second part, we evaluate different ablation patterns and compare the results with the standard Maze III. In the third part, the effect of incomplete standard Maze III procedure is studied and in the fourth part, a comparison between clinical data and computer simulations is presented.

\section{METHODS}

\section{Biophysical Atrial Model}

Several biophysical models of human atria have been developed, with different tradeoffs between accuracy of anatomical representation and computational load..$^{6,9,22}$ Elaborate atrial models already published take into account conduction bundles and evaluate normal propagation or atrial arrhythmias with simulations lasting a few seconds. ${ }^{6,9,22}$ For example, Harrild et al. developed an 
anatomically accurate three-dimensional model of human atria, used for the simulation of sinus rhythm. ${ }^{9}$ Vigmond et al. developed a model of canine atria $(3.3 \mathrm{~cm}$ wide) including Bachmann's bundle, crista terminalis and pectinate muscles. ${ }^{22}$ Since our objective is to simulate a high number of different AF ablation patterns in real size human atria over a time interval sufficient to assess the effectiveness of AF termination (each simulation lasting up to $30 \mathrm{~s}$ ), our AF biophysical model is simplified as much as possible to keep computational load tractable.

As atrial geometry plays an important role during arrhythmia, ${ }^{1}$ our biophysical model is based on sliced magnetic resonance images of human atria. It is meshed with triangular elements. ${ }^{25}$ The resulting triangulated, smooth surface consists of 100,000 triangles $(50,000$ nodes $)$ with an average edge length of $\Delta l=0.6 \mathrm{~mm}$. A finer mesh ( 100,000 nodes, $\Delta l=0.4 \mathrm{~mm}$ ) was used to check the accuracy and to ensure the absence of artifacts. At each node, a cellular model was implemented and the conductivity between them was equal, leading so to a homogeneous and isotropic surface. Furthermore, the thickness of the atrial wall is discarded (monolayer model). The structure contains holes representing the entries and exits of the major vessels as well as the valves to the ventricles (see Fig. 1 for main anatomical features). The cellular membrane model used is the Luo-Rudy model based on six ionic currents. ${ }^{14}$ This model, originally developed to represent the electrical activity of ventricles, was chosen in order to reduce computational load. To approximate the specific electrical properties of atrial cells, action potential duration (APD) was adapted as published in Virag et al. ${ }^{23}$ by adjusting channel conductances $G_{N a}$ to $16 \mathrm{mS} / \mathrm{cm}^{2}, G_{K}$ to $0.423 \mathrm{mS} / \mathrm{cm}^{2}$ and $G_{S i}$ to $0.055 \mathrm{mS} / \mathrm{cm}^{2}$. As a result, baseline APD is equal to $170 \mathrm{~ms}$ and APD restitution curve is flattened (with a maximal slope smaller than one), which is in agreement with data for experimentally remodeled atria cells. ${ }^{13}$ The electrical propagation is solved in a monodomain cardiac tissue as described in Virag et al. ${ }^{23}$
In the present study, a tradeoff was made between model complexity and computational load in such a way to allow us to simulate the initiation of several arrhythmias such as atrial flutter and sustained AF, as well as subsequent ablation procedures. We have also developed a more sophisticated version of our model using the Courtemanche atrial membrane kinetics ${ }^{3}$ including anisotropy, heterogeneities and the computation of electrograms, at the expense of substantially increased computational load. ${ }^{10}$ Validation of the model so far was based on comparisons with human and animal experiments and measurements of atrial electrophysiology. ${ }^{10,23}$

\section{Ablation Patterns}

Ablation lines are therapeutic interventions that can be easily simulated in a biophysical model of AF. ${ }^{5}$ In our model, they are simulated by setting the conductivity tensor to zero between the cardiac cells located on both sides of the lines, thus creating ideal ablation lines, defined as continuous and transmural (monolayer surface). This is equivalent to a surgical ablation line or to an RF ablation on both the epicardial and endocardial sides. To implement an ablation line into our geometry, two nodes of the triangular mesh are selected ( $\mathrm{S}$ and $\mathrm{E}$ in Fig. 2(A)). A principal vector $(\overrightarrow{S E})$ is constructed between the starting node $(\mathrm{S})$ and the end node (E). Vectors $\left(\overrightarrow{S N}_{i}\right)$ from the starting node to its neighboring nodes $\left(\mathrm{N}_{i}\right)$ are computed. Each vector is then projected onto the principal vector $(\overrightarrow{S E})$ and the neighbor with the largest projection value is selected as the new starting node $\left(S^{\prime}\right)$ (Fig. 2(B)). The principal vector is then updated with this new node (Fig. 2(C)) and the procedure is repeated until the end node is reached. Finally, the nodes that are selected during the iteration are part of the ablation line and their conductivity to their neighbors are set to zero (dashed lines in Fig. 2(D)).

In this work, the gold standard Maze III procedure represented in Fig. 3 was taken as the reference. ${ }^{4,20}$ This pattern

\section{Anterior view Posterior view}

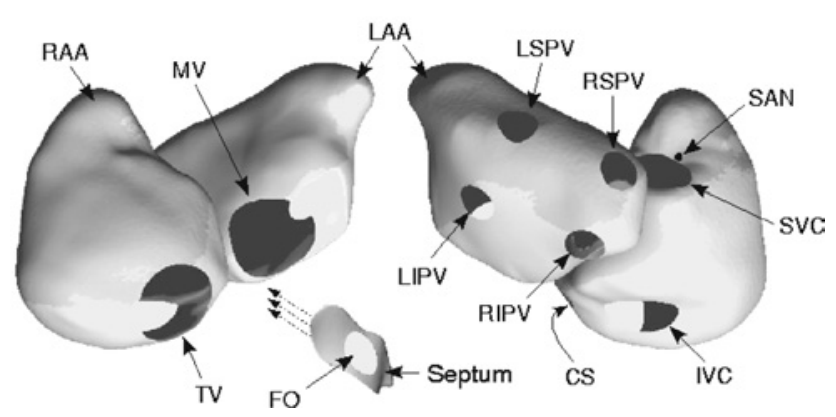

FIGURE 1. Geometry of human atria based on MR images. The left/right atrium appendages are indicated by LAA/RAA. The major vessels and valves are included: Tricuspid valve (TV), mitral valve (MV), inferior vena cava (IVC), superior vena cava (SVC), pulmonary veins (LSPV, RSPV, LIPV and RIPV), coronary sinus (CS) and fossa ovalis (FO). The sino-atrial node (SAN) is represented by a dot. 
(A)

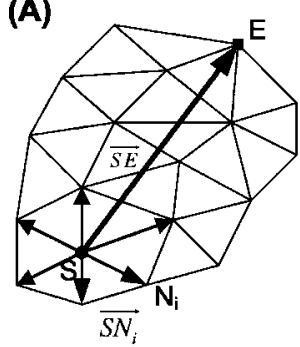

(B)

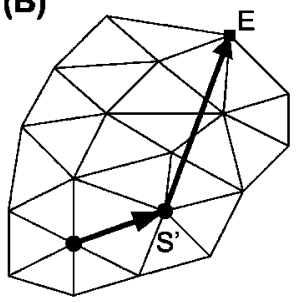

(C)

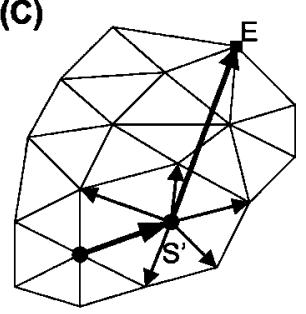

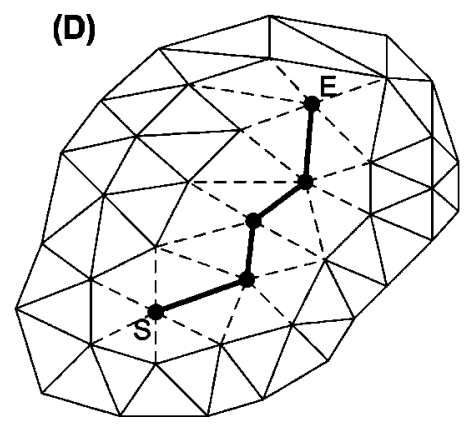

FIGURE 2. Creation of an ablation line in the computer model. (A) Selection of starting node and end node. (B) Update of the starting node. (C) Selection of neighbor node. (D) Nodes on the ablation line.

results in the highest long term success rate in clinical applications. Eleven other, less invasive, patterns (Fig. 4) were also evaluated. Among these, some reflect the most commonly used in the clinic. The first row in Fig. 4 involves right atrial ablation lines only: a single ablation line through the isthmus between the inferior vena cava (IVC) and the tricuspid valve (TV) (Fig. 4(A)), a single ablation line between the superior vena cava (SVC) and IVC (Fig. 4(B)), and a combination of the two previous patterns (Fig. 4(C)). Two ablation patterns are limited to the left atrium: ablation lines connecting all four pulmonary veins (Fig. 4(D)), and ablation lines completely isolating the pulmonary veins (Fig. 4(H)). The six remaining ablation patterns involve

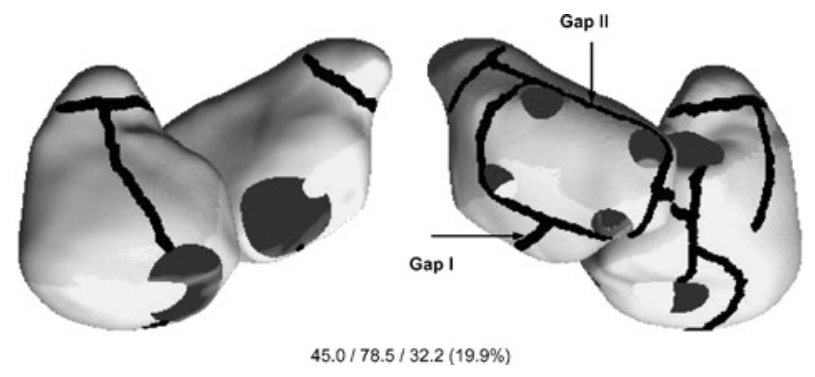

FIGURE 3. Standard Maze III procedure: ablation lines are indicated by dark lines. Gaps (for incomplete procedure only) are introduced along the ablation lines indicated by arrows. Gap1 and Gap 2 are positioned at several locations on the line and have a width of $1.3,1.7,2.4$ or $3.0 \mathrm{~mm}$. The ablations length (cm) / the total boundary due to ablation and perimeters of natural obstacles such as veins and valves $(\mathrm{cm}) /$ the isolated tissue surface $\left(\mathrm{cm}^{2}\right)$ (percentage) are indicated. both atria (Figs. 4(E)-4(G) and Figs. 4(I)-4(K)). The estimated total lengths of the ablation lines, approximated by the Euclidian distance between nodes arbitrarily placed along the line, ranges from $1 \mathrm{~cm}$ for the simplest pattern (Fig. 4(A)) to $45 \mathrm{~cm}$ for the Maze III pattern of Fig. 3. The ablation patterns on the third line in Fig. 4 include a complete isolation of the pulmonary vein region, implying that no electrical activity can be propagated from the pulmonary veins to the atria. The isolated area is $11.5 \mathrm{~cm}^{2}$, i.e. $7.2 \%$ of the whole atrial tissue. For the Maze III procedure of Fig. 3, the isolated area is $32.2 \mathrm{~cm}^{2}$, i.e. $19.9 \%$ of the whole atrial tissue.

In clinical procedures, RF ablation lines are sometimes applied only to the endocardial side and the ablation lines may be incomplete, introducing gaps allowing atypical flutter or fibrillation recurrence. To reproduce these phenomena, discontinuities on the Maze III ablation patterns were simulated. The first discontinuity (Gap I in Fig. 3) was placed along the ablation line connecting the pulmonary veins and the mitral valve annulus and the second (Gap II in Fig. 3) along the ablation line connecting the upper pulmonary veins. Four gap widths $(1.3,1.7,2.4$, and $3.0 \mathrm{~mm})$ were simulated in the middle of the ablation line and the widest gap was also tested at both extremities of the ablation line. This protocol allows us to study the effect of gap width and position separately with a minimum number of simulations.

Further simulations were performed to compare the results obtained in our model with clinical data. The comparison is based on a simplified Maze III procedure using RF ablation (Mini-Maze, Fig. 5(A)). This pattern was 
Left line only

Right line only

Rightine only
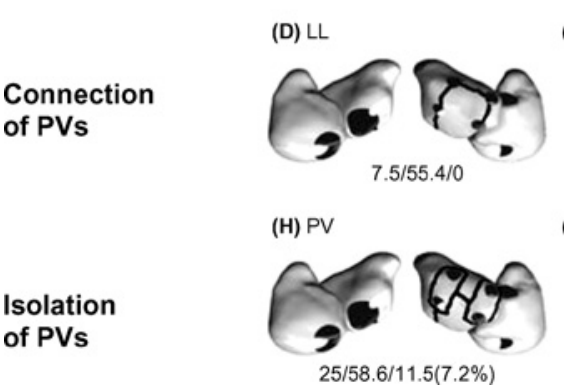

(E) $L L+1 O$

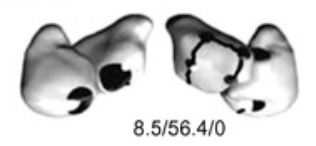

IO line

(A) 10

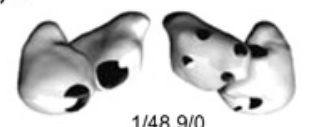

(l) $\mathrm{PV}+1 \mathrm{O}$

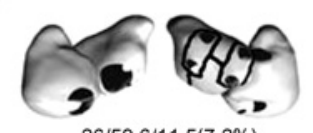

26/59.6/11.5(7.2\%)
SIVC line

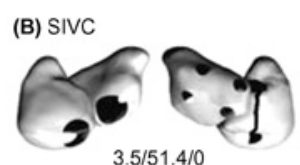

(F) LL+SIVC

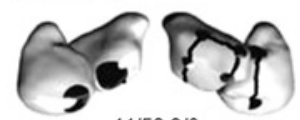

$11 / 58.9 / 0$

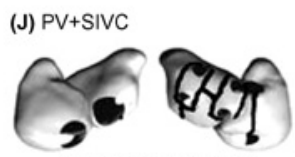

28.5/62.1/11.5(7.2\%)
IO+SIVC lines

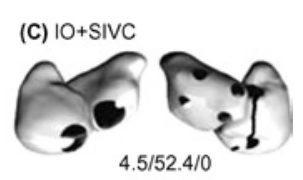

(G) $\mathrm{LL}+1 \mathrm{O}+\mathrm{SIVC}$

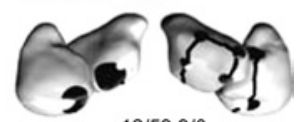

$12 / 59.9 / 0$

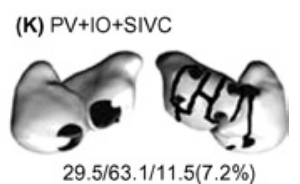

FIGURE 4. Tested ablation patterns (black lines). Under each pattern, the ablations length (cm) / the total boundary due to ablation and perimeters of natural obstacles such as veins and valves $(\mathrm{cm}) /$ the isolated tissue surface $\left(\mathrm{cm}^{2}\right)($ percentage) are indicated. (A) Isthmus only, IO. (B) Superior to inferior vena cava line, SIVC. (C) IO+SIVC. (D) Left line only (connection of pulmonary veins), LL. (E) LL+IO. (F) LL+SIVC (G) LL+IO+SIVC. (H) Pulmonary veins isolation, PV. (I) PV+IO, PV+IO. (J) PV+SIVC. (K) PV+IO+SIVC. The first row/column shows patterns with right/left lines only. The total number of ablation lines is increased from left to right and from top to bottom.

implemented in our biophysical model after its application in 46 patients with permanent drug refractory AF. ${ }^{19}$ The RF left atrial compartmentalization was performed during mitral valve repair or replacement in which the patients were divided into two groups: Group I with endocardial RF application only (20 patients) and Group II with both endoand epicardial RF application (26 patients). Both groups of patients were similar in terms of age, AF duration and left atrial size. Group II was modeled using the Mini-Maze of
Fig. 5(A) with perfect ablation lines, while Group I was simulated in the biophysical model with a gap width of $3 \mathrm{~mm}$ on the left isthmus to mimic non-transmurality of the ablation.

\section{Initiation of $A F$ and Application of Ablation Patterns}

AF was induced using burst pacing near the sino-atrial node region (SAN). Burst pacing was chosen because it
(A) Mini-Maze

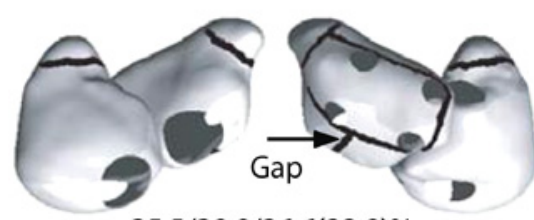

$35.5 / 38.8 / 36.6(22.8) \%$
(C) Mini-Maze ABBI
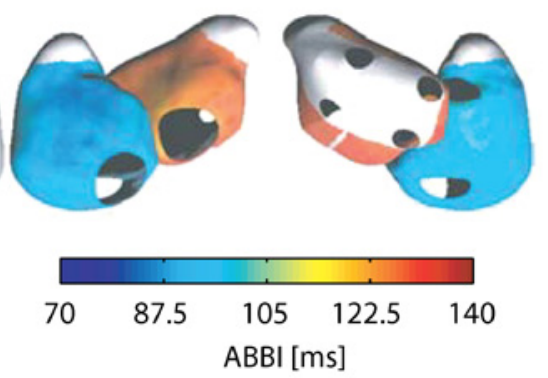

(B) Results

\begin{tabular}{lcccc}
\hline $\begin{array}{l}\text { Ablation } \\
\text { Pattern }\end{array}$ & $\begin{array}{c}\text { SR } \\
\text { (Clinical/Computer) }\end{array}$ & $\begin{array}{c}\text { AF } \\
\text { (Clinical / Computer) }\end{array}$ & $\begin{array}{c}\text { AFL } \\
\text { (Clinical / Computer) }\end{array}$ & p \\
\hline Group I & $12(60 \%) / 13(65 \%)$ & $4(20 \%) / 2(10 \%)$ & $4(20 \%) / 5(25 \%)$ & 0.66 \\
Group II & $24(92 \%) / 23(88 \%)$ & $2(8 \%) / 3(12 \%)$ & $0 / 0$ & 0.64 \\
\hline
\end{tabular}

FIGURE 5. Comparison between computer simulations and clinical experiments. (A) Mini-Maze pattern. Position of the gap is indicated for Group I patients. (B) Distribution of the average beat-to-beat intervals (ABBI) for Group II patients. (C) Result from clinical data and computer simulations. 
does not require any adjustment, timing or location of ectopic beats, a procedure that may be time consuming due to the high sensitivity of the system to these parameters; in other words there is a critical time window for AF initiation in this model. ${ }^{10}$ The SAN region was paced at a frequency of $20 \mathrm{~Hz}$. The wave breaks leading to $\mathrm{AF}$ occurred through the combined effect of non-uniform geometry and dynamical dispersion of refractoriness. After $3 \mathrm{~s}$, pacing was stopped and the system evolved freely. In all cases, simulated sustained AF lasting longer than 5 min was observed as several independent wavelets traveling randomly throughout the tissue. AF was perpetuated through functional and anatomical reentries, namely, fronttail interactions and collisions with anatomical obstacles. Following simulated sustained $\mathrm{AF}$ ( $10 \mathrm{~s}$ after the beginning of burst pacing), several time instants were selected at intervals of $1 \mathrm{~s}$ for the application of the different ablation patterns. Because of the random nature of AF, these time instants reflect moments of AF having different parameters such as the number of wavelets, etc. The different ablation patterns were applied instantaneously, after which the time to AF termination (TAFT) was documented. If no termination was found to occur within $30 \mathrm{~s}$ the ablation was defined as unsuccessful. This process was repeated for each of the studied ablation patterns.

For the Maze III procedure (Fig. 3), a total of 118 simulations were performed with time instants selected successively during sustained AF. For each of the other 11 ablation patterns (Fig. 4(A)-4(K)), 20 simulations were performed using the first 20 time instants used for the Maze III protocol. The 118 time instants selected for the Maze III procedure were also used to simulate the discontinuities in this pattern (gaps as shown in Fig. 3). Finally, 20 and 26 time instants respectively were selected to simulate the Mini-Maze ablation in Group I and Group II (Fig. 5). In total, 1800 simulations of ablation were performed with a TAFT ranging from 0.25 to $30 \mathrm{~s}$. The computation time is approximately $1 \mathrm{~h}$ for a $1 \mathrm{~s}$ simulation on a standard PC (Pentium-III 1.4 GHz).

\section{Measure of Organization}

We characterized the dynamics of AF using two variables: (1) the percentage of excited tissue, i.e. the percentage of nodes with an action potential above a fixed threshold $(-60 \mathrm{mV}), 2)$ the number of wavelets, defined as the number of connected regions of excited tissue. In our simulation, during sustained AF the percentage of excited tissue evolved within a range from $25.3 \%$ to $72.2 \%$, and the number of wavelets varied between 1 and 11 . The correlation coefficient between these two variables was -0.53 , indicating that they evolved in opposite directions.

To evaluate the effect of each ablation pattern, the average beat-to-beat interval (ABBI) was computed at each node of the mesh immediately after the ablation pattern was applied. For each ablation pattern, ABBI values were computed for the simulation having the highest TAFT or for an unsuccessful termination. This leads to distribution maps of ABBI on the surface of the atria. A small ABBI value means the presence of small reentrant circuits or high fibrillatory activity whereas a large ABBI value is the expression of larger circuits with the highest probability of transition into sinus rhythm.

\section{RESULTS}

\section{Maze III Procedure}

All 118 simulations of the standard Maze III procedure successfully stopped AF, with a mean TAFT of $1.3 \mathrm{~s} \pm 0.8 \mathrm{~s}$. Fig. 6 shows the impact of the time instants selected (the percentage of excited tissue and the number of wavelets) for the application of the ablation pattern on the TAFT. This figure suggests that there is no significant correlation between a successful ablation and specific time instants of AF. Thus, a single isolated wavelet unblocked by the ablation lines may generate or maintain sustained AF. Conversely, an initial state with several wavelets may rapidly lead to a short TAFT value because these wavelets can block or annihilate each other.

\section{Comparison of Ablations Patterns}

The percentages of successful conversions for the Maze III procedure of Fig. 3 as well as for the 11 ablation patterns described in Fig. 4, are summarized in Table 1. They are ordered by decreasing success rate. Four groups could be distinguished: (1) a group with a $100 \%$ success rate, (2) a group with a high success rate from $80 \%$ to $95 \%$, (3) a group with an intermediate success rate from $60 \%$ to $65 \%$, and (4) a group with a low success rate from $20 \%$ to $25 \%$. The more complex the ablation patterns, the lower the TAFT values. It should be noted that there is a large standard deviation in TAFT values for all ablation patterns. The best result with the shortest TAFT is obtained by the standard Maze III procedure.

The corresponding distributions of ABBI are shown in Fig. 7 and are color coded: ABBI values ranged between $70 \mathrm{~ms}$ (in blue) and $140 \mathrm{~ms}$ (in red) corresponding to frequencies of $14.3 \mathrm{~Hz}$ and $7.1 \mathrm{~Hz}$, respectively. The ablation patterns with a minimum number of lines led to low values of ABBI, indicating no decrease in the rate of the AF. The ablation patterns isolating the pulmonary veins (Figs. $7(\mathrm{H})-7(\mathrm{~K})$ ) led to a higher left atrial ABBI (>100 ms) indicating longer intervals between activations. Only the standard Maze III procedure resulted in a high ABBI in both atria.

\section{Discontinuous Maze III Procedure}

The results of simulations of discontinuities in the Maze III are listed in Table 2. The success rates obtained with 

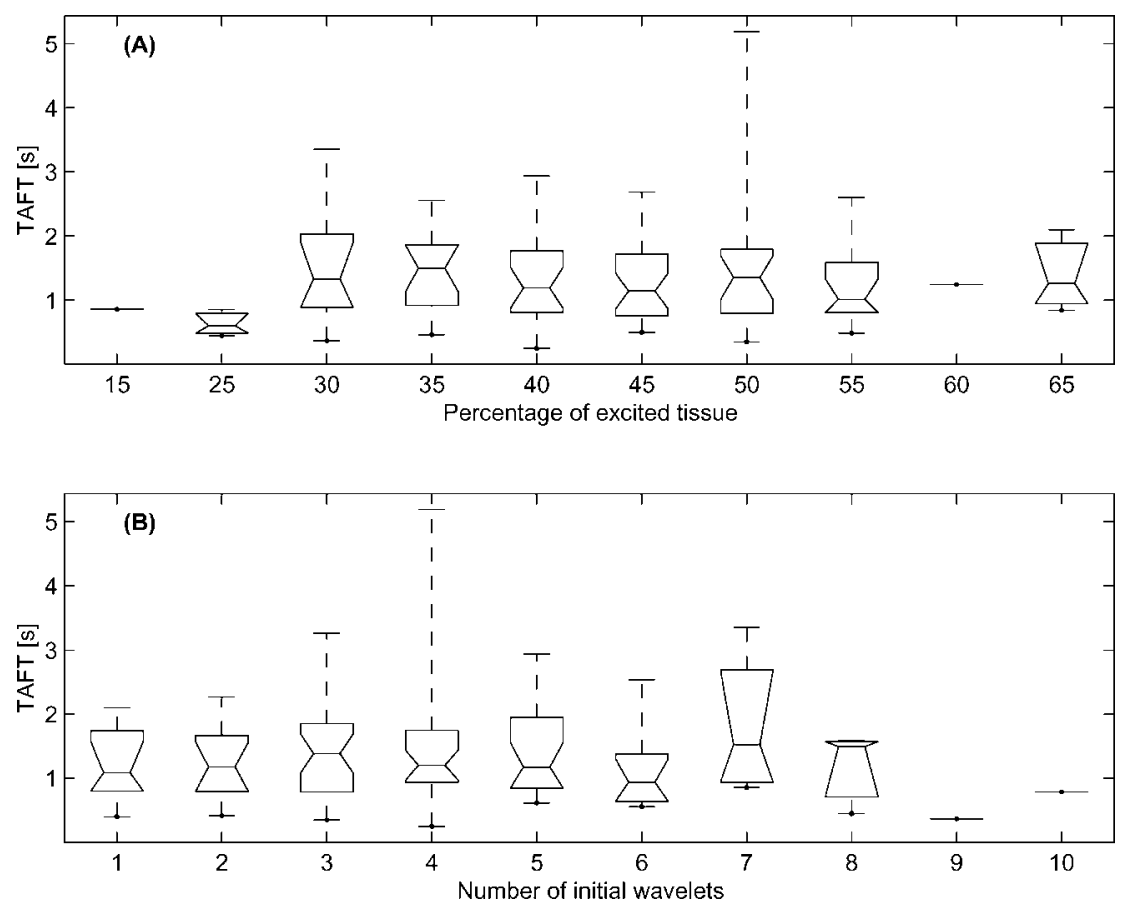

FIGURE 6. (A) TAFT vs. percentage of excited tissue. (B) TAFT vs. number of initial wavelets. The boxes have lines at the lower quartile, median, and upper quartile values. The whiskers are lines extending from each end of the box to show the range of the data.

the gap in the ablation line between the upper pulmonary veins (Gap II in Fig. 3) are similar to those of the Maze III $(100 \%)$. The activation maps showed that wavelets passing through this gap were further blocked by the other ablation lines connecting the four pulmonary veins. Therefore, the position or size of this gap was not critical for the success of AF termination and the means TAFT were similar. With the gap between the pulmonary veins and mitral valve annulus (Gap I in Fig. 3) the success rates were affected by both width and location. The effect of the gap location was a

TABLE 1. Efficiency of different ablation patterns: Mean time to AF termination and percentages of successful termination.

\begin{tabular}{lcc}
\hline Ablation patterns & $\begin{array}{c}\text { Mean termination } \\
\text { time } \pm \text { SD (s) }\end{array}$ & $\begin{array}{c}\text { Successful } \\
\text { termination (\%) }\end{array}$ \\
\hline Maze III & $1.3 \pm 0.8$ & 100 \\
PV+IO+SIVC (K) & $6.0 \pm 5.0$ & 100 \\
LL+SIVC (F) & $7.0 \pm 5.4$ & 100 \\
LL+IO+SIVC (G) & $9.8 \pm 6.6$ & 100 \\
PV+IO (I) & $7.8 \pm 7.2$ & 95 \\
LL+IO (E) & $8.8 \pm 5.4$ & 90 \\
PV+SIVC (J) & $10.1 \pm 4.5$ & 80 \\
PV (H) & $15.8 \pm 9.8$ & 65 \\
LL (D) & $11.9 \pm 8.5$ & 60 \\
IO+SIVC (C) & $16.3 \pm 8.8$ & 60 \\
SIVC (B) & $15.1 \pm 11.6$ & 25 \\
IO (A) & $16.2 \pm 3.4$ & 20 \\
\hline
\end{tabular}

decrease in success rate as the gap was moved towards the mitral annulus. Furthermore, the wider the gap (except for a gap value of $1.3 \mathrm{~mm}$ which has no effect), the lower the termination rate. In both cases the decrease of the success rate was linked to the presence of an uncommon flutter around the mitral valve.

\section{Mini-Maze and Comparison to Clinical Data}

Results obtained with the Mini-Maze for both the biophysical model and the clinical experiments are summarized in Fig. 5(B). For Group II (with perfect ablation lines in the biophysical model and both endo- and epicardial RF ablation in the clinic) the percentage of conversion to sinus rhythm is $88 \%$ (with simulations) and $92 \%$ (clinical data), which is slightly lower than for the complete Maze III. For Group I (with a discontinuous ablation line in the biophysical model and only an endocardial RF ablation in the clinic) this percentage was found to be smaller and atypical left atrial flutter was observed both in the biophysical model and in patients. No significant difference was observed between the clinical data and the computer results. Fig. 5(C) represents the corresponding ABBI distribution.

\section{DISCUSSION}

In this paper, a biophysical model of human atria was used to evaluate different patterns of AF ablation. In the first part, the gold standard Maze III procedure was evaluated 
Maze III

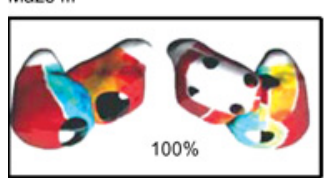

(D) LL

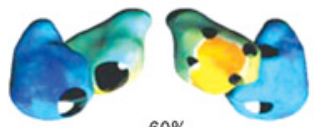

$60 \%$

(H) PV

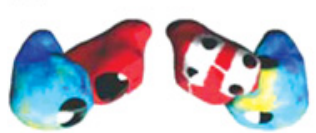

$65 \%$
(A) 10

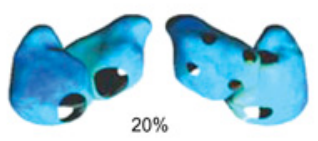

(E) $\mathrm{LL}+10$

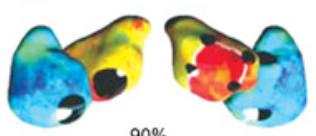

$90 \%$

(I) $\mathrm{PV}+10$

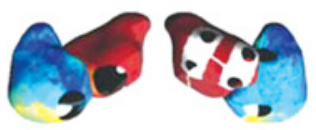

$95 \%$
(B) SIVC

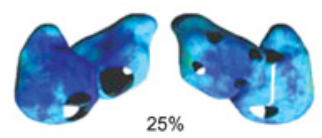

(F) LL+SIVC

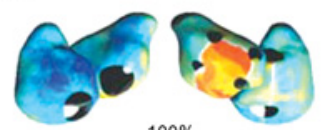

$100 \%$

(J) PV+SIVC

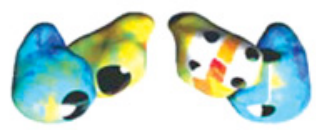

$80 \%$
(C) IO+SIVC

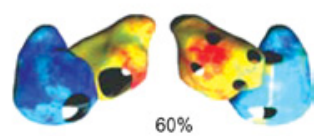

(G) $\mathrm{LL}+\mathrm{IO}+\mathrm{SIVC}$

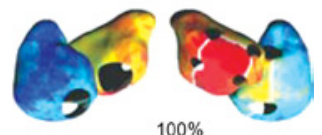

(K) $\mathrm{PV}+\mathrm{IO}+\mathrm{SIVC}$

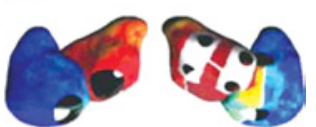

$100 \%$

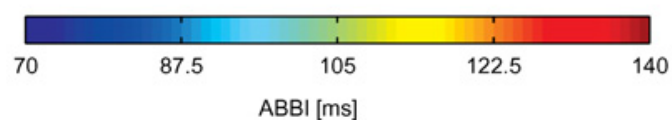

FIGURE 7. Percentage of success rate and distributions of the average beat-to-beat intervals (ABBI) for each of the 12 ablation patterns studied (ablation lines and electrically isolated regions are resented in white). The gold standard Maze III is placed at the top left of the figure. (A) Isthmus only, IO. (B) Superior to inferior vena cava line, SIVC. (C) IO+SIVC. (D) Left line only (connection of pulmonary veins), LL. (E) LL+IO. (F) LL+SIVC (G) LL+IO+SIVC. (H) Pulmonary veins isolation, PV. (I) PV+IO, PV+IO. (J) PV+SIVC. (K) PV+IO+SIVC.

using different time instants of AF. In the second part, several ablation patterns implying right and/or left atrium as well as isolation of pulmonary veins were performed and compared to the Maze III procedure. In the third part, the effect of some discontinuities in the Maze III ablation pattern was assessed. Finally, the results obtained with the biophysical model were compared to clinical data obtained from patients suffering from permanent $\mathrm{AF}$ and undergoing a RF limited maze ablation.

\section{Comparison of Ablation Patterns}

Among the simulated ablation patterns, the most efficient is the Maze III procedure with a success rate of $100 \%$ and the smallest mean TAFT value of $1.3 \pm 0.8 \mathrm{~s}$. This ablation pattern is able to terminate AF independently of the initial states in the tissue at the moment of application of the lines. The success rate obtained is in agreement with clinical data, where the complete surgical Maze III pattern had the highest long-term success rate ranging from 80 to $99 \% .^{4,7,12,20}$ Limitations of the surgical Maze are the necessitation for open-heart surgery, the time-consuming nature of the procedure and the related complications. RF ablation represents a less invasive alternative, however the surgical Maze procedure is technically difficult to reproduce using $\mathrm{RF}$ due to frequent discontinuities in the ablation lines, especially if ablation is performed from the endocardial side only. Our simulation of discontinuities in the Maze

TABLE 2. Efficiency of discontinuities in the Maze III: Mean time to AF termination and percentages of successful termination.

\begin{tabular}{|c|c|c|c|c|c|c|}
\hline & \multicolumn{3}{|c|}{ Position gap I } & \multicolumn{3}{|c|}{ Position gap II } \\
\hline & $\begin{array}{l}\text { Mean termination } \\
\text { time } \pm \mathrm{SD}(\mathrm{s})\end{array}$ & $\begin{array}{l}\text { Successful } \\
\text { termination (\%) }\end{array}$ & $\begin{array}{l}\text { Flutter around } \\
\text { MV (\%) }\end{array}$ & $\begin{array}{l}\text { Mean termination } \\
\text { time } \pm \mathrm{SD}(\mathrm{s})\end{array}$ & $\begin{array}{c}\text { Successful } \\
\text { termination (\%) }\end{array}$ & $\mathrm{AF}(\%)$ \\
\hline \multicolumn{7}{|c|}{ Gap widths at the middle position } \\
\hline Width: $1.3 \mathrm{~mm}$ & $1.4 \pm 0.8$ & 100 & 0 & $1.4 \pm 0.6$ & 100 & 0 \\
\hline Width: $1.7 \mathrm{~mm}$ & $1.4 \pm 0.9$ & 89.0 & 11.0 & $1.4 \pm 0.9$ & 100 & 0 \\
\hline Width: $2.4 \mathrm{~mm}$ & $1.4 \pm 0.8$ & 82.2 & 17.8 & $1.4 \pm 0.8$ & 100 & 0 \\
\hline Width: $3.0 \mathrm{~mm}$ & $1.4 \pm 0.8$ & 79.7 & 20.3 & $1.4 \pm 0.7$ & 100 & 0 \\
\hline \multicolumn{7}{|c|}{ Gap positions with $3 \mathrm{~mm}$ width } \\
\hline Up or left & $1.4 \pm 0.8$ & 79.7 & 20.3 & $1.4 \pm 0.7$ & 100 & 0 \\
\hline Middle & $1.4 \pm 0.8$ & 79.7 & 20.3 & $1.4 \pm 0.7$ & 100 & 0 \\
\hline Down or right & $1.3 \pm 0.7$ & 72.0 & 28.0 & $1.4 \pm 0.7$ & 100 & 0 \\
\hline
\end{tabular}


III showed that a significant decrease in success rate could indeed be observed depending on both gap location and size.

Research on ablation patterns is directed towards finding possible ways of reproducing the success of the complete Maze procedure while using minimal ablation lines. We have tested here the efficacy of several patterns. As expected, the ablation pattern with only a $1 \mathrm{~cm}$ right isthmus line (IO in Fig. 7 (A)) was the least efficient, with a success rate of $20 \%$ and many wavelets still present on both atria after ablation. This limited pattern originally intended to cure atrial flutter ${ }^{2}$ has very little effect on the more complex dynamics of AF, as shown in the distribution of ABBI with low values ranging from 80 to $90 \mathrm{~ms}$ (Fig. 7(A)) indicating the absence of slowing down in atrial activity. The second least effective pattern is the one with an ablation line between both venae cavae (SIVC in Fig. 7(B)). This pattern is not commonly used in clinical interventions but is presented here as part of a systematic study. It led to a success rate of $25 \%$ and a distribution of ABBI slightly different from the previous pattern, namely with $\mathrm{ABBI}$ values lower than 80 $\mathrm{ms}$ in the region around the pulmonary veins and the right atrial free wall (Fig. 7(B)).

If we exclude the simplified IO and SIVC patterns leading to a very low success rate, we can identify three groups in the results obtained with our biophysical model (Table 1). The first group has a success rate ranging from 60 to $65 \%$ and implies ablation patterns with lines only in the right or only in the left atrium. The first pattern in this group is a combination of IO+SIVC with a total ablation length of $4.5 \mathrm{~cm}$ (Fig. 7(C)). Interestingly, this pattern involving right atrial lines only significantly modified the distribution of ABBI in the left atrium with values $>120 \mathrm{~ms}$ indicating slower activity (Fig. 7(C)). This type of pattern has been tested in a clinical study by Garg et al ${ }^{7}$ to determine whether lesions could be applied only in the right atrium to suppress the risk associated with catheter ablation in the left atrium. They reported that AF was suppressed on the long-term in $67 \%$ of the patients following right atrial compartmentalization, which may be compared to the $60 \%$ we obtained. Two other patterns implying lines in the left atrium only led to similar results in terms of success rate and ABBI distribution: the LL pattern (Fig. 7(D)) with a total ablation length of $7.5 \mathrm{~cm}$ and the PV pattern with a full isolation of the pulmonary veins (Fig. 7(H)). These patterns led to a success rate of $60 \%$ and $65 \%$ respectively. The isolation of the pulmonary veins did not affect the success rate considerably, whereas some differences in the ABBI values in the left atrium could be observed ( $>130 \mathrm{~ms}$ for the PV pattern and $<120 \mathrm{~ms}$ for the LL pattern). The success rate obtained with the biophysical model for these patterns shows some contradiction with clinical experiments of ablation in the region of the pulmonary veins reporting a long-term success rate of $82 \% .{ }^{12}$ This difference may be partly explained by the fact that our model of AF is based on multiple reentrant wavelets (similar to a permanent AF in the clinical setting implying a diseased atrial tissue), which is very different to the focal AF due to ectopic beats (atrial foci) generally located in the pulmonary veins and acting as triggers for $\mathrm{AF}^{8}$ (and closer to a paroxysmal form of AF in the clinical setting with a surrounding healthy atrial tissue).

The results obtained in the first group of ablation patterns confirmed the fact that lines in the right or in the left atrium only are not sufficient to isolate all multi-wavelets re-entries. We noticed that ablation lines in the right atrium could impact the ABBI distribution in the left atrium, while on the other hand left lines had little effect on wavelet reentry in the right atrium.

We identified a second group with a success rate ranging from 80 to $95 \%$. All patterns in this group are based on the LL or the PV pattern with one ablation line added in the right atrium (the IO or the SIVC line). The addition of the SIVC line to the PV pattern (Fig. 7(J)) increased the success rate from $65 \%$ to $80 \%$, while the addition of the IO line to the same PV pattern (Fig. 7(I)) increased the success rate from $65 \%$ to $95 \%$. Similarly, by adding the IO line to the LL pattern (Fig. 7(E)), the success rate increased from $60 \%$ to $90 \%$. However, for all 3 patterns in this group, the ABBI distribution showed no significant reduction of the activity in the right atrium.

Our third group of ablation patterns shows a success rate comparable to the Maze III procedure. The best approaches are the LL pattern with an SIVC line (Fig. 7(F)) and the LL or PV patterns with both an IO and an SIVC line (Figs. 7(G) and $7(\mathrm{~K})$ ). They both lead to a $100 \%$ success rate. Interestingly, for all these successful patterns the ABBI values were lower in the right atrium as compared to the Maze III, suggesting the persistence of residual wavelets and reentries not affecting termination. The easiest pattern to implement and, furthermore, with the shortest total ablation length is the LL+SIVC pattern. However, as mentioned earlier, our model does not take into account focal AF with triggers in the pulmonary veins. Therefore in clinical experiments, patterns without full isolation of the pulmonary veins could lead to worse results.

\section{Effect of Imperfect Ablation Lines}

In addition to the simulation of alternative solutions to the full Maze III procedure, we also simulated the effect of discontinuities in this ablation pattern. Gaps with a different size and location were simulated for two lines of, this pattern. Our results show that a gap, whatever its size or location, placed between the upper pulmonary veins, has no effect on the success rate (Gap II in Table 2). When the mechanism of AF is self-perpetuation based on multiple reentries, only three lines out of four are needed for the pulmonary vein connections. This phenomenon is confirmed by the high success rate observed in simulations 
with patterns LL+SIVC and LL+IO+SIVC (Figs. 7(F)$7(\mathrm{G})$ ) which include only three lines (without gap) between the pulmonary veins. By looking at the activation maps, we observed that some wavelets still passing through this gap were further block by ablation lines. On the other hand, a gap positioned along the ablation line connecting the pulmonary veins and the mitral annulus (Gap I in Table 2) can significantly decrease the success rate due to a flutter around the mitral valve (a phenomenon already clinically observed $^{11}$ ). This decrease in success rate is more important for gaps close to the mitral annulus since the presence of flutter is facilitated. Success rate is also decreased as the gap width is increased from 1.7 to $3 \mathrm{~mm}$. This suggests that for small gaps, conduction blocks may occur, therefore preserving the effect of the ablation line. If no conduction block is associated with the gap, atrial flutter will occur. Mapping studies in animals indicate that gaps smaller than $5 \mathrm{~mm}$ in ablation lines may result in conduction blocks. ${ }^{15}$

\section{Comparison of Simulations with Clinical Data}

We compared the results obtained in our biophysical model with clinical experiments (Fig. 5) for two groups of patients: Group I Mini-Maze with a gap in the left isthmus to mimic non-transmurality (RF application on one side only of the atrium) and Group II with a complete MiniMaze. The clinical results show that Group I patients have a significantly lower success rate (60\%) compared to the Group II patients (92\%), highlighting the importance of transmurality of the ablation pattern. These data, as well as the presence of left atrial flutter for Group I patients, are confirmed by our biophysical model, and no significant statistical difference was observed between clinical and computer data. Fig. 5(C) shows the ABBI distribution for the Group II. When compared to the full Maze III, the Mini-Maze procedure, despite right atrial appendage removal, leads to $\mathrm{ABBI}$ values close to $80 \mathrm{~ms}$ in the right atrium, signifying persistent wavelet activity. The ABBI distribution is very similar to those obtained with the other ablation pattern also implying an isolation of the pulmonary veins (Fig. 7(H)-7(K)).

\section{Limitations}

Finally, even though the results obtained with our atrial model are consistent with clinical observations, we should highlight some of its limitations. In our study, the number of simulations performed with the standard Maze III procedure is identical to that of patients in the literature, i.e. 118 patients. ${ }^{4,20}$ However, a direct comparison between our computer simulations and clinical data is difficult, since the geometry of our biophysical model was one and the same during the whole experiment, whereas the atrial anatomy differs in each patient. Furthermore, in contrast to surgical operations, during computer simulations all elements of the ablation lines were applied instantaneously. Moreover, when simulating non-transmural ablation lines, we introduced one gap of arbitrary width in a line of the biophysical model, while during clinical endocardial ablation many gaps of unknown size can be present. We should also highlight the fact that our model of AF takes into account reentrant wavelets only and cannot be used in the case of rapid foci. For instance, if a rapid focus appears in the pulmonary vein region, incomplete ablation lines around pulmonary veins (Fig. 3 Gap II) will not necessarily prevent AF reinitiation. Finally, in order to keep computation time in a tractable range, we used a simplified atrial model. Our single layer cell model does not include anisotropic fast-conducting regions, fiber orientation, crista terminalis and pectinate muscles, which may all play a role during $\mathrm{AF}^{6,16} \mathrm{~A}$ version of our model incorporating some of these aspects has been developed ${ }^{3}$ at the expense of an increased complexity and computational load. The inclusion of such details needs further validated data.

\section{CONCLUSIONS}

This paper shows how a computer model of human atria can be used to compare existing surgical or RF ablation procedures and to study the effect of imperfect ablation lines. The results of our simulated ablation lines confirm the good performance of the gold standard Maze III procedure, and suggest that the development of less invasive patterns needs to include ablation lines in both the right and the left atrium. It has also been observed in our simulations that some lines are more sensitive to imperfections (for example due to non-transmurality of ablation) than others, leading generally to the presence of uncommon atrial flutter after ablation, a phenomenon also observed in the clinic. A comparison of our simulation data with clinical data shows no significant difference in the case of patients suffering from permanent AF. These results indicate that, through an interaction with clinicians involved in surgical procedures, biophysical models are promising tools for the development of new ablation patterns.

\section{ACKNOWLEDGMENTS}

This study was made possible by grants from the TheoRossi-Di-Montelera Foundation, Medtronic Europe, the Swiss Governmental Commission of Innovative Technologies (CTI), and the Swiss National Science Foundation (SNSF). The authors wish to thank Ryan Lahm, Drs. Josée Morisette and Arthur Stillman who kindly furnished the atrial geometry surface model. The authors also would like to thank Prof. Adriaan van Oosterom for helpful discussions and suggestions. 


\section{REFERENCES}

${ }^{1}$ Allessie, M., P. L. Rensma, J. Brugada, J. L. R. M. Smeets, O. Penn, and C. J. H. J. Kirchhof. Pathophysiology of atrial fibrillation. In: Cardiac Electrophysiology: From Cell to Beside, edited by D. P. Zipes and J. Jalife. Philadelphia: W. B. Saunders Co, 1990 , pp. 548-559.

${ }^{2}$ Cosio F. G., F. Arribas, M. Lopez-Gil, and H. D. Gonzalez. Radiofrequency ablation of atrial flutter. J. Cardiovasc. Electrophysiol. 7:60-70, 1996.

${ }^{3}$ Courtemanche M., R. J. Ramirez, and S. Nattel. Ionic mechanisms underlying human atrial action potential properties: Insights from a mathematical model. Am. J. Physiol. 275:H301H321, 1998.

${ }^{4}$ Cox J. L., R. B. Schuessler, H. J. Jr. D'Agostino, C. M. Stone, B.-C. Chang, M. E. Cain, P. B. Corr, and J. P. Boineau. The surgical treatment of atrial fibrillation: III. Development of a definitive surgical procedure. J. Thorac. Cardiovasc. Surg. 101:569583,1991

${ }^{5}$ Ellis W. S., A. SippensGroenewegen, and M. Lesh. The effect of linear lesions on atrial defibrillation threshold and spontaneous termination. A computer model study. PACE 20:1145,] 1997.

${ }^{6}$ Ellis, W. S., A. SippensGroenewegen, D. M. Auslander, and M. D. Lesh. The role of the crista terminalis in atrial flutter and fibrillation: A computer modeling study. Ann. Biomed. Eng. 28:742-54, 2000.

${ }^{7}$ Garg A., W. Finneran, M. Mollerus, U. Birgersdotter-Green, O. Fujimura, L. Tone, and G. K. Feld. Right atrial compartmentalization using radiofrequency catheter ablation for management of patients with refractory atrial fibrillation. J. Thorac. Cardiovasc. Surg. 10:763-771, 1999.

${ }^{8}$ Haissaguerre M., P. Jais, D. Shah, A. Takahashi, M. Hocini, G. Quiniou, S. Garrigue, A. Le Mouroux, P. Le Métayer, and J. Clémenty. Spontaneous initiation of atrial fibrillation by ectopic beats originating in the pulmonary veins. New Eng. J. Med. 339:649-666, 1998.

${ }^{9}$ Harrild, D. M., and C. S. Henriquez. A computer model of normal conduction in the human atria. Circ. Res. 87:25e-36e, 2000.

${ }^{10}$ Jacquemet V., N. Virag, Z. Ihara, L. Dang, O. Blanc, S. Zozor, J.-M. Vesin, L. Kappenberger, and C. Henriquez. Study of unipolar electrogram morphology in a computer model of atrial fibrillation. J. Cardiovasc. Electrophysiol. 14:S172-S179, 2003.

${ }^{11}$ Jais, P., D. C. Shah, M. Haissaguerre, M. Hocini, J. T. Peng, A. Takahashi, S. Garrigue, P. Le Metayer, and J. Clementy. Mapping and ablation of left atrial flutters. Circulation, 101(25):2928-2934, 2000.

${ }^{12}$ Kottkamp H., G. Hindricks, D. Hammel, R. Autschbach, J. Mergenthaler, M. Borggrefe, G. Breithardt, F. W. Mohr, and H.
H. Scheld. Intraoperative radiofrequency ablation of chronic atrial fibrillation: A left curative approach by elimination of anatomic "anchor" reentrant circuit. J. Cardiovasc. Electrophysiol. 10:772-780, 1999.

${ }^{13}$ Li D., L. Zhang, J. Kneller, and S. Nattel. Potential ionic mechanism for repolarization differences between canine right and left atrium. Circ. Res. 88:1168-1175, 2001.

${ }^{14}$ Luo C.-H., and Y. Rudy. A model of the ventricular cardiac action potential. Circ. Res. 68:1501-1526, 1991.

${ }^{15}$ Mitchell M. A., T. H. Everett, H. Li, J. M. Mangrum, and D. E. Haines. Morphological and physiological characteristics of discontinuous linear atrial ablations during atrial pacing and atrial fibrillation., J. Cardiovasc. Electrophysiol. 10(3):378-386, 1999.

${ }^{16}$ Nakagawa H., R. Lazzara, T. Khastgir, K. J. Beckman, J. H. McClelland, S. Imai, J. V. Pitha, A. E. Becker, M. Arruda, M. D. Gonzalez, L. E. Widman, M. Rome, J. Neuhauser, X. Wang, J. D. Calame, M. D. Goudeau, and W. M. Jackman. Role of the tricuspid annulus and the eustachian valve/ridge on atrial flutter. Relevance to catheter ablation of the septal isthmus and a new technique for rapid identification of ablation success. Circulation 94:407-24, 1996.

${ }^{17}$ Ninio D. M. Contemporary management of atrial fibrillation. Aust. Prescr. 23:100-102, 2000.

${ }^{18}$ Petrac D. Electrical therapy of atrial fibrillation. J. Clin. Basic Cardiol. 4:123-129, 2001.

${ }^{19}$ Ruchat, P., J. Schaepfer, A. Delabays, M. Hurni, J. Milne, and L. K. von Segesser. Left atrial radiofrequency compartmentalization for chronic atrial fibrillation during heart surgery. Thorac. Cardiovasc. Surg. 50:155-159, 2002.

${ }^{20}$ Sie H. T., W. P. Beukema, A. Elvan, and A. R. Misier. New strategies in the surgical treatment of atrial fibrillation. Cardiovasc. Res. 58:501-509, 2003.

${ }^{21}$ Surawicz B. Brief history of cardiac arrhythmias since the end of the nineteenth century: Part II. J. Cardiovasc. Electrophysiol. 15:101-111, 2004

${ }^{22}$ Vigmond, E. J., R. Ruckdeschel, and N. Trayanova. Reentry in a morphologically realistic atrial model. J. Cardiovasc. Electrophysiol. 12:1046-1054, 2001.

${ }^{23}$ Virag N., V. Jacquemet, C. S. Henriquez, S. Zozor, O. Blanc, J.-M. Vesin, E. Pruvot, and L. Kappenberger. Study of atrial arrhythmias in a computer model based on MR images of human atria. Chaos 12:754-763, 2002.

${ }^{24}$ Wellens H. J. Contemporary management of atrial flutter. Circulation 106:649-652, 2002.

${ }^{25}$ Zozor S., O. Blanc, V. Jacquemet, N. Virag, J.-M. Vesin, E. Pruvot, L. Kappenberger, and C. S. Henriquez. A numerical scheme for modeling wavefront propagation on a monolayer of arbitrary geometry. IEEE Trans. Biomed. Eng. 50:412-420, 2003. 